Published in final edited form as:

Gynecol Oncol. 2020 January ; 156(1): 70-76. doi:10.1016/j.ygyno.2019.10.005.

\title{
SENTINEL LYMPH NODE MAPPING ALONE COMPARED TO MORE EXTENSIVE LYMPHADENECTOMY IN PATIENTS WITH UTERINE SEROUS CARCINOMA
}

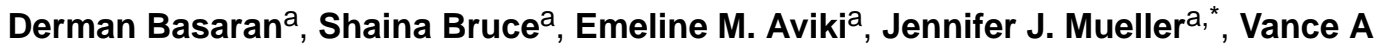

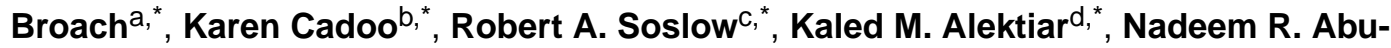 \\ Rustuma, ${ }^{a}{ }^{\star}$, Mario M. Leitao Jr. ${ }^{a,{ }^{*}}$ \\ aDepartment of Surgery, Memorial Sloan Kettering Cancer Center, New York, NY 10065 \\ bDepartment of Medicine, Memorial Sloan Kettering Cancer Center, New York, NY 10065 \\ 'Department of Pathology, Memorial Sloan Kettering Cancer Center, New York, NY 10065 \\ dDepartment of Radiation Oncology, Memorial Sloan Kettering Cancer Center, New York, NY \\ 10065
}

*Weill Cornell Medical College, New York, NY 10065

\begin{abstract}
Objectives-The objective of our study was to assess survival among patients with uterine serous carcinoma (USC) undergoing sentinel lymph node (SLN) mapping alone versus patients undergoing systematic lymphadenectomy (LND).
\end{abstract}

\footnotetext{
Correspondence: Mario M. Leitao, Jr., MD, Gynecology Service, Department of Surgery, Memorial Sloan Kettering Cancer Center, 1275 York Avenue, New York, NY 10065, Phone: (212) 639-3987, leitaom@ @skcc.org.

AUTHOR CONTRIBUTIONS

Derman Basaran: Concept and design; collection of data; analysis and interpretation of data; drafting manuscript; revising manuscript; final approval of manuscript

Shaina Bruce: Collection of data; drafting manuscript; revising manuscript; final approval of manuscript Emeline M. Aviki: Analysis and interpretation of data; revising manuscript; final approval of manuscript Jennifer J. Mueller: Analysis and interpretation of data; revising manuscript; final approval of manuscript Vance A. Broach: Analysis and interpretation of data; revising manuscript; final approval of manuscript Karen Cadoo: Analysis and interpretation of data; revising manuscript; final approval of manuscript

Robert A. Soslow: Analysis and interpretation of data; revising manuscript; final approval of manuscript

Kaled M. Alektiar: Analysis and interpretation of data; revising manuscript; final approval of manuscript

Nadeem R. Abu-Rustum: Concept and design; analysis and interpretation of data; revising manuscript; final approval of manuscript Mario M. Leitao, Jr:: Concept and design; analysis and interpretation of data; writing manuscript; revising manuscript; final approval of manuscript

CONFLICT OF INTEREST STATEMENT: None of the authors declare any conflicts of interest.

DISCLOSURES

Dr. Abu-Rustum reports grants from Stryker/Novadaq, grants from Olympus, grants from GRAIL, outside the submitted work.

Dr. Cadoo reports other* from Astra Zeneca, other** from Syndax Pharmaceuticals, outside the submitted work.

(*Travel, accommodation, meal; institutional support for therapeutic trial; **institutional support for therapeutic trial)

Dr. Soslow reports personal fees from Ebix/Oakstone*; personal fees from Cambridge University Press**; personal fees from Springer Publishers**; personal fees from Roche***, outside the submitted work.

(*Preparation of recorded lectures; **royalties; ***one lecture)

Publisher's Disclaimer: This is a PDF file of an unedited manuscript that has been accepted for publication. As a service to our customers we are providing this early version of the manuscript. The manuscript will undergo copyediting, typesetting, and review of the resulting proof before it is published in its final form. Please note that during the production process errors may be discovered which could affect the content, and all legal disclaimers that apply to the journal pertain.
} 
Methods-We retrospectively reviewed patients undergoing primary surgical treatment for newly diagnosed USC at our institution from 1/1/1996-12/31/2017. Patients were assigned to either SLN mapping alone (SLN cohort) or systematic LND without SLN mapping (LND cohort).

Progression-free (PFS) and overall survival (OS) were estimated using Kaplan-Meier method, compared using Logrank test.

Results—245 patients were available for analysis: 79 (32.2\%) underwent SLN, 166 (67.7\%) LND. 132 (79.5\%) in the LND cohort had paraaortic LND (PALND) versus none in the SLN cohort. Median age: 66 and 68 years in the SLN and LND cohorts, respectively ( $>0.05)$. Proportion of stage I/II disease: $67.1 \%(\mathrm{n}=53)$ and $64.5 \%(\mathrm{n}=107)$ in the SLN and LND cohorts, respectively (p>0.05). Median follow-up: 23 (range, 1-96) and 66 months (range, 4-265) in the SLN and LND cohorts, respectively ( $\mathrm{p}<0.001)$. Two-year OS in stage I/II disease $(\mathrm{n}=160,60.1 \%)$ : 96.6\% (SE+/-3.4) and 89.6\% (SE+/-2.2) in the SLN and LND cohorts, respectively ( $\mathrm{p}=0.8$ ). Twoyear OS in stage III disease ( $\mathrm{n}=77): 73.6 \%(\mathrm{SE}+/-10.2)$ and $77.3 \%(\mathrm{SE}+/-5.8)$ in the SLN and LND cohorts, respectively $(\mathrm{p}=0.8)$.

Conclusions-SLN mapping alone and systematic LND yielded similar survival outcomes in stage I-III USC. In our practice, the SLN algorithm has replaced systematic LND as the primary staging modality in this setting.

\section{Keywords}

Endometrial cancer; Sentinel lymph node; Uterine serous carcinoma; Lymphadenectomy

\section{INTRODUCTION}

Endometrial cancer is the most common gynecologic malignancy. Uterine serous carcinoma (USC) is an aggressive histologic subtype of this disease [1]. USCs comprise approximately $10 \%$ of all new cases of endometrial cancers diagnosed annually, but account for $40 \%$ of endometrial cancer deaths [2]. USC tends to spread beyond the uterus. Because of this tumor's aggressive behavior, adjuvant treatment and primary surgical staging are required for most patients [2].

As with other endometrial cancers, the initial staging surgery for USC includes retroperitoneal lymph node assessment, to ascertain disease prognosis and examine the factors associated with oncologic outcomes [3]. Although randomized trials have failed to show any survival benefit associated with systematic nodal dissection in endometrial cancer, detection of nodal disease does affect staging, prognosis and possibly postoperative treatment decisions. However, because of its low histologic prevalence, USC is underrepresented in randomized studies. The morbidity associated with nodal dissection-including lymphedema, lymphocyst formation, and other potential organ injuries-is considerable [4-7]; therefore, questions have been raised regarding the necessity for extensive lymphadenectomy (LND) in every case.

Sentinel lymph node (SLN) mapping has been proposed as an alternative method for detecting nodal metastases in endometrial cancer. Use of the Memorial Sloan Kettering 
Cancer Center SLN algorithm results in a very low $(<0.5 \%)$ false-negative predictive value (FNPV) in the detection of nodal metastases $[3,8]$.

The published studies to date that have addressed the use of SLN mapping in USCs have primarily included other histologic subtypes of endometrial cancer, such as grade 3 endometrioid tumors, clear cell carcinomas and carcinosarcomas, and the applicability of those results to a purely USC population is limited. In other studies, patients who underwent SLN and full lymphadenectomy (LND) are combined [9-12]. The impact of the SLN algorithm on oncologic outcomes in USC is, therefore, less well-established. In the current study, we evaluated SLN mapping in patients with USC by comparing the outcomes of those who underwent SLN mapping alone (i.e., with no additional non-SLN nodes removed) to those who underwent pelvic +/- paraaortic LND without SLN mapping.

\section{METHODS}

After obtaining approval from the Institutional Review Board at Memorial Sloan Kettering Cancer Center (MSK), we retrospectively reviewed the records of all patients who underwent surgical staging for USC at MSK from January 1, 1996 through December 31, 2017. Surgical staging included total hysterectomy (irrespective of surgical/anatomic route), removal of adnexa, and lymph node evaluation using systematic LND and/or the SLN algorithm. The decision to proceed with systematic LND or SLN mapping was made by the surgeon. Expert gynecologic pathology review was performed on all cases.

SLN mapping was performed according to our previously published institutional protocol [8]. Patients typically had $4 \mathrm{~mL}$ of either blue dye or indocyanine green (ICG) injected into the cervix at the 3 and 9 o'clock positions. Retroperitoneal evaluation involved the localization of dye-filled lymphatic channels extending from the parametria to the primary nodal basins, and subsequent removal of nodes with uptake of dye/ICG. Pathologic analysis included ultrastaging of all SLNs in 5-micrometer $(\mu \mathrm{m})$ sections at each of two levels, 50- $\mu \mathrm{m}$ apart. Anti-cytokeratin AE1:AE3 (Ventana Medical Systems Inc., Tucson, AZ) was used if the initial hematoxylin and eosin (H\&E) sections were negative. Nodal disease was classified as macro-metastases, micro-metastases, or isolated tumor cells.

Our institutional SLN algorithm includes routine peritoneal and serosal evaluation and peritoneal washings. This is followed by retroperitoneal evaluation, removal of all mapped SLNs and any additional lymph nodes suspicious for disease, without performing routine intra-operative frozen section consultation. In the current study, completion LND was not performed unless indicated by the algorithm. In cases where failure in mapping was noted in a hemipelvis, a side-specific LND was performed. Further para-aortic dissection was completed at the discretion of the attending surgeon, with the guidance of preoperative imaging.

At our institution a combination of chemotherapy and radiation therapy (RT) is typically offered to patients who have both early myoinvasive and advanced stage USC. Decisions for adjuvant therapy were not changed nor modified based on whether LND or SLN mapping alone was done. However, the final decision regarding adjuvant therapy is made through 
consultation between patients and their primary providers at MSK. Follow-up visits are scheduled every 3 months for the first 2-3 years, and then every 6 months for the next 2-3 years. Surveillance via computerized axial tomography scans is typically performed every 3-6 months during the first 2 years, and then every 6-12 months for the next 2-3 years. Patterns of recurrence are categorized as local/regional (including disease recurrence at the vaginal cuff and/or pelvis), isolated nodal, and distant/multifocal spread.

Clinical, pathologic and follow-up data were reviewed for each patient. Descriptive statistics and association tests were performed using SPSS software (v. 23; SPSS Inc., Chicago, IL). The Kruskal-Wallis test was used to compare continuous variables, and Fisher's exact test or the Chi-squared test used to compare categorical variables. Progression-free survival (PFS) was calculated from the date of surgery to the date of progression, date of death, or date of last follow-up. Overall survival (OS) was calculated from the date of surgery to the date of death or date of last follow-up. OS and PFS estimates were obtained using the Kaplan-Meier method, and the Logrank test was used to compare cohorts. A Cox proportional hazards model was used to determine independent prognostic factors for survival. All calculated pvalues were two-sided, and $\mathrm{p}$-values $<0.05$ were considered statistically significant.

\section{RESULTS}

Four-hundred two patients with USC who received treatment at our institution between January 1, 1996 and December 31, 2017 were identified. After excluding 5 patients (1 who had neoadjuvant CT prior to surgery, 3 with history of multiple primary malignancies, and 1 for serous endometrial intraepithelial carcinoma but no invasive disease), 397 patients were available for the final analysis. Thirty-seven of these patients were excluded because they had failed SLN mapping on one side of the pelvis and had undergone unilateral side-specific completion LND; 114 were excluded because they had had SLN mapping and additional non-SLNs removed. Seventy-nine (32.2\%) patients underwent SLN mapping with no additional lymph node dissection (SLN cohort) and 280 (67.8\%) patients underwent bilateral lymph node dissection (LND cohort).

Clinical and pathologic features of the study patients are presented in Table 1. Median age was 66 years (range, 51-89) in the SLN cohort and 68 years (range, 44-87) in the LND cohort ( $p>0.05$ ). There was no difference in median body mass index (BMI) between the cohorts. Both cohorts had similar stage distribution, with stage I/II disease being the most common. The rates of deep myometrial invasion, lymphovascular space invasion (LVSI), and positive peritoneal washings were similar in both patient cohorts $(\mathrm{p}>0.05$ for all observations). The median number of excised lymph nodes was 18.5 (range:1-75) in the LND cohort and 3 (range:1-11) in the SLN cohort ( $\mathrm{p}<0.001$ ). Paraaortic lymph node dissection was performed in 132 (79.5\%) patients in the LND cohort and no patients in the SLN cohort $(\mathrm{P}<0.001)$. Rates of nodal metastasis were $26.5 \%$ and $29.5 \%$ in the SLN and LND cohorts, respectively ( $\mathrm{p}=0.6)$.

The majority of patients received postoperative adjuvant treatment $(84.8 \%$ and $89.8 \%$ in the SLN and LND cohorts, respectively; $\mathrm{p}=0.9)$. Chemotherapy alone or combined with RT was 
the most common adjuvant treatment in both cohorts. Carboplatin plus paclitaxel was the most commonly administered chemotherapeutic agent.

The median follow-up time was 23 months (range,1-96) for patients in the SLN cohort, and 66 months (range, 4-265) for patients in the LND cohort ( $<<0.001)$. Recurrence was observed in $97(39.5 \%)$ patients, and recurrence rates were similar in both of the study cohorts $(\mathrm{p}=0.5)$. Distant/multifocal disease recurrence was predominant in both of the study cohorts. Patterns of disease recurrence are described in Table 2. The 2-year PFS rates were $58.8 \%(\mathrm{SE} \pm 6.5)$ and $64.9 \%$ (SE \pm 3.8$)$ in the SLN and LND cohorts, respectively $(\mathrm{p}=0.478$ ) (Figure 1). On univariate analysis, patients with advanced stage disease, deep myometrial invasion, LVSI, positive washings, and those who did not undergo para-aortic nodal dissection had significantly shorter PFS. On multivariate analysis, deep myometrial invasion, positive washings, LVSI, and advanced stage disease were independent prognostic factors for worse PFS (Table 3).

At the time of analysis, 78 (31.8\%) of 245 study patients were deceased. The 2-year OS rates were $89.1 \%(\mathrm{SE} \pm 4.3)$ in the SLN cohort and 83.9\% (SE \pm 2.9$)$ in the LND cohort $(\mathrm{p}=0.9)$ (Figure 2). Univariate analysis showed that stage III disease, $250 \%$ myometrial invasion, LVSI, or positive washings were associated with significantly lower 2-year OS $(\mathrm{p}<0.001)$. On multivariate analysis, positive peritoneal cytology (HR: 2.11 ; 95\% CI [1.243.61]; $\mathrm{p}=0.006$ ), >50\% myometrial invasion (HR:1.82; 95\% CI [1.02-3.22)] and LVSI (HR: 2.04; 95\% CI [1.18-3.51]; $\mathrm{p}=0.01)$ were independent prognostic factors for diminished OS (Table 4).

Patients who underwent para-aortic nodal dissection had higher 2-year PFS rates $(69.7 \%$ [SE $\pm 4.1]$ vs. 55.4.3\% [SE \pm 5.2$] ; \mathrm{p}=0.015$ ) on univariate analysis, but multivariate analysis failed to show any significant association between para-aortic nodal dissection and 2- year PFS (HR 1.19; 95\% CI [0.97-2.29], $\mathrm{p}=0.06$ ). Additionally, para-aortic nodal dissection did not demonstrate an effect on 2-year OS in patients with USC (86.8\% [ \pm 2.4$]$ vs. 83.2 [ \pm 2.7$]$, $\mathrm{p}=0.12$ ).

\section{DISCUSSION}

In 1988, the International Federation of Gynecology and Obstetrics (FIGO) changed the staging system for endometrial cancer from clinical staging to surgical staging, following publication of the seminal paper by Creasman et al. [13, 14]. Two large randomized European trials failed to demonstrate any survival benefit for routine pelvic lymph node dissection, but showed higher rates of lymphedema in the LND arms [4, 5]. SLN mapping in patients with endometrial cancer started in the early 2000s and has become increasingly utilized over the past decade [3]. MSK's institutional SLN mapping algorithm (which indicates side-specific pelvic LND when an SLN is not detected during mapping) significantly decreased the false-negative detection rate from $15 \%$ to $2 \%$.Most importantly, it decreased the FNPV to $0.4 \%$ in women with low-risk endometrial cancer [8]. The multicenter, prospective FIRES trial provided robust data regarding the accuracy of SLN mapping in patients with endometrial cancer, suggesting that SLN mapping has a high 
degree of diagnostic accuracy in detecting endometrial cancer metastases and can safely replace LND in the staging of endometrial cancer. [10].

We previously compared the efficacy and oncologic outcomes of SLN mapping and selective lymph node dissection for determining lymphatic spread in patients with low-risk and highrisk endometrioid tumors, including those with high-risk histology (including USC) [15-17]. The largest published series on SLN mapping in patients with USC included 248 cases from our institution [12]. We noted 2-year PFS rates of 77\% (95\% CI, 68-83\%) in the SLN group and $71 \%(95 \% \mathrm{CI}, 61-79 \%)$ in the LND group ( $\mathrm{p}=0.3)$. However, in that analysis, our objective was to examine how incorporation of SLN mapping affected oncologic outcomes. In that analysis, the criteria for inclusion in the SLN cohort was significantly broader, as many patients in the "SLN cohort" also underwent either planned backup or selective LND due to failed SLN mapping. The current study was designed to evaluate the oncologic outcomes of patients with USC who only had SLNs removed and did not undergo additional LND. In the current study, therefore, the median total number of removed nodes was only 3 in the SLN cohort, compared to 12 in our previous analysis.

Several investigators have described their experience with SLN mapping in terms of nodal disease detection and survival in patients with high-risk endometrial cancer $[9,11,12,18$, 19]. Soliman et al. reported the results of a prospective trial of 123 patients with high-risk endometrial cancer (grade 3 endometrioid, clear cell, USC) who underwent SLN mapping followed by complete LND [11]. The bilateral SLN detection rate was only $58 \%$, but $95 \%$ of $23(23 \%)$ node-positive women were correctly identified as having disease in the SLN, resulting in an false-negative rate of 5\% and a FNPV of $0.8 \%$. (In our study, the bilateral mapping rate in the SLN cohort was 89.9\%, with nodal metastasis identified in 21 (26.5\%) patients.) Another retrospective study by Touhami et al. of 128 patients with high-risk endometrial cancer reported an SLN mapping sensitivity of 95.8\% and NPV of 98.2\% [19]. Both the prospective and retrospective studies mentioned above added routine lymph node dissection after SLN mapping, but each of these studies included fewer than 50 cases of USC. A recent European multi-institutional retrospective analysis compared the SLN algorithm versus SLN with selective LND in high-risk, early-stage endometrial cancer. This study showed that 5-year DFS rates were similar in both groups: 79.2\% (CI 95\%: 56-91) for patients in the SLN algorithm group, and 81.6\% (CI 95\%: 67-90) for patients in the SLN with LND group ( $\mathrm{p}=0.831$ ) [9]. Although this study has a methodology very similar to ours, most of the patients had high-risk endometrioid endometrial cancer and there were a limited number of patients with USC. Thus, as would be expected, the study reported better DFS rates than were found in our patient cohort.

Some retrospective data suggest that patients with high-risk endometrial cancer may benefit from para-aortic nodal dissection, despite randomized trials in all risk categories demonstrating no survival advantage $[4,5,20,21]$. The SEPAL study suggested that pelvic and para-aortic LND was associated with better oncologic outcomes in high-risk patients compared with pelvic LND alone; however, as in most studies, the number of patients with non-endometrioid histology was very small $(\mathrm{n}=55,8 \%)$, which limits our ability to gather true value in USC specifically [20]. Most recently, Venigalla etal. used the National Cancer Database (NCDB) to analyze 7250 patients with clinical stage I serous carcinoma, clear cell 
carcinoma, or carcinosarcoma who underwent hysterectomy from 2010 to 2013 [21]. Pelvic LND was associated with decreased risk of death compared with no LND, in all histologic subtypes (HR $=0.65,95 \% \mathrm{CI}$ : 0.59-0.71). The addition of para-aortic LND was associated with a decreased risk of death $(\mathrm{HR}=0.85,95 \% \mathrm{CI}: 0.79-0.91)$ compared to pelvic LND alone for patients with USC. It is unclear from the NCDB analyses whether LND was done only in the setting of microscopic nodal disease, or whether nodal status impacted postoperative therapy decisions; furthermore, as the NCDB has no central pathology review, we cannot ascertain the accuracy of pathologic assessments. In our study, none of the patients in the SLN cohort had a para-aortic LND. However, we observed similar 2-year OS rates for the patients in the LND cohort, most of whom underwent para-aortic LND. Our results do not appear to support the theory that the removal of microscopic nodal disease results in a survival benefit, as the randomized trials had suggested. The lymphatic channels do not end at either the inferior mesenteric or the renal veins. It is highly likely that one would encounter nodal disease in the lymphatics above the renal vein in cases with infrarenal nodal metastasis. Therefore, LND to remove microscopic disease would have to be carried above the renal veins, and likely to the scalene nodal basins, in order to be truly therapeutic.

The current study has limitations common to all retrospective studies. In addition, the median follow-up time was shorter in our SLN cohort than LND cohort (23 months vs. 66 months), and this impacted the outcome analysis as we chose to report 2-year outcomes. Our study included patients treated at our institution during a long period of time, but it is important to note that patient management has changed over time. Additional follow-up on the outcomes of SLN mapping is required. Nevertheless, our findings suggest that SLN mapping alone is safe in patients with USC. Furthermore, SLN mapping alone can be utilized to evaluate nodal spread while avoiding the morbidity associated with systematic nodal dissection.

\section{ACKNOWLEDGEMENTS}

Funding: This study was funded in part through the NIH/NCI Support Grant P30 CA008748.

\section{REFERENCES}

1. Siegel RL, Miller KD, Jemal A. Cancer statistics, 2016. CA Cancer J Clin 2016;.66:7-30. doi: 10.3322/caac.21332. [PubMed: 26742998]

2. Moore KN, Fader AN. Uterine papillary serous carcinoma. Clin Obstet Gynecol 2011; 54:278-291. doi: 10.1097/GRF.0b013e318218c755. [PubMed: 21508697]

3. Holloway RW, Abu-Rustum NR, Backes FJ, Boggess JF, Gotlieb WH, Jeffrey Lowery W, et al. Sentinel lymph node mapping and staging in endometrial cancer: A Society of Gynecologic Oncology literature review with consensus recommendations. Gynecol Oncol 2017; 146:405-415. doi: 10.1016/j.ygyno.2017.05.027. [PubMed: 28566221]

4. ASTEC study group; Kitchener H, Swart AM, Qian Q, Amos C, Parmar MK. Efficacy of systematic pelvic lymphadenectomy in endometrial cancer (MRC ASTEC trial): a randomised study. Lancet 2009; 373:125-136. doi: 10.1016/S0140-6736(08)61766-3. [PubMed: 19070889]

5. Benedetti Panici P, Basile S, Maneschi F, Alberto Lissoni A, Signorelli M, Scambia G, et al. Systematic pelvic lymphadenectomy vs. no lymphadenectomy in early-stage endometrial carcinoma: randomized clinical trial. J Natl Cancer Inst 2008; 100:1707-1716. doi: 10.1093/jnci/ djn397. [PubMed: 19033573]

Gynecol Oncol. Author manuscript; available in PMC 2021 January 01. 
6. Abu-Rustum NR, Alektiar K, Iasonos A, Lev G, Sonoda Y, Aghajanian C, et al. The incidence of symptomatic lower-extremity lymphedema following treatment of uterine corpus malignancies: a 12-year experience at Memorial Sloan-Kettering Cancer Center. Gynecol Oncol 2006; 103(2): 714 718. doi: 10.1016/j.ygyno.2006.03.055. [PubMed: 16740298]

7. Dowdy SC, Borah BJ, Bakkum-Gamez JN, Weaver AL, McGree ME, Haas LR, et al. Prospective assessment of survival, morbidity, and cost associated with lymphadenectomy in low-risk endometrial cancer. Gynecol Oncol 2012; 127:5-10. doi: 10.1016/j.ygyno.2012.06.035. [PubMed: 22771890]

8. Barlin JN, Khoury-Collado F, Kim CH, Leitao MM Jr, Chi DS, Sonoda Y, et al. The importance of applying a sentinel lymph node mapping algorithm in endometrial cancer staging: beyond removal of blue nodes. Gynecol Oncol 2012; 125:531-535. doi: 10.1016/j.ygyno.2012.02.021. [PubMed: 22366409]

9. Buda A, Gasparri ML, Puppo A, Mereu L, De Ponti E, Di Martino G, et al. Lymph node evaluation in high-risk early stage endometrial cancer: A multi-institutional retrospective analysis comparing the sentinel lymph node (SLN) algorithm and SLN with selective lymphadenectomy. Gynecol Oncol 2018; 150:261-266. doi: 10.1016/j.ygyno.2018.06.003. Epub 2018 Jun 7. [PubMed: 29887483]

10. Rossi EC, Kowalski LD, Scalici J, Cantrell L, Schuler K, Hanna RK, et al. A comparison of sentinel lymph node biopsy to lymphadenectomy for endometrial cancer staging (FIRES trial): a multicentre, prospective, cohort study. Lancet Oncol 2017; 18:384-392. doi: 10.1016/ S1470-2045(17)30068-2. [PubMed: 28159465]

11. Soliman PT, Westin SN, Dioun S, Sun CC, Euscher E, Munsell MF, et al. A prospective validation study of sentinel lymph node mapping for high-risk endometrial cancer. Gynecol Oncol 2017; 146):234-239. doi: 10.1016/j.ygyno.2017.05.016. [PubMed: 28528918]

12. Schiavone MB, Scelzo C, Straight C, Zhou Q, Alektiar KM, Makker V, et al. Survival of Patients with Serous Uterine Carcinoma Undergoing Sentinel Lymph Node Mapping. Ann Surg Oncol 2017; 24:1965-1971. doi: 10.1245/s10434-017-5816-4. [PubMed: 28258415]

13. Creasman WT, Morrow CP, Bundy BN, Homesley HD, Graham JE, Heller PB. Surgical pathologic spread patterns of endometrial cancer. A Gynecologic Oncology Group Study. Cancer 1987; 60(8 Suppl): 2035-2041. doi: 10.1002/1097-0142(19901015)60:8+<2035::aid-cncr2820601515>3.0.co; 2-8. [PubMed: 3652025]

14. Mikuta JJ. International Federation of Gynecology and Obstetrics staging of endometrial cancer 1988. Cancer 1993; 71(4 Suppl):1460-1463. doi: 10.1002/cncr.2820710409. [PubMed: 8431880]

15. Zahl Eriksson AG, Ducie J, Ali N, McGree ME, Weaver AL, Bogani G, et al. Comparison of a sentinel lymph node and a selective lymphadenectomy algorithm in patients with endometrioid endometrial carcinoma and limited myometrial invasion. Gynecol Oncol 2016; 140:394-399. doi: 10.1016/j.ygyno.2015.12.028. [PubMed: 26747778]

16. Ducie JA, Eriksson AGZ, Ali N, McGree ME, Weaver AL, Bogani G, et al. Comparison of a sentinel lymph node mapping algorithm and comprehensive lymphadenectomy in the detection of stage IIIC endometrial carcinoma at higher risk for nodal disease. Gynecol Oncol 2017; 147:541548. doi: 10.1016/j.ygyno.2017.09.030. [PubMed: 28965698]

17. Schlappe BA, Weaver AL, Ducie JA, Eriksson AGZ, Dowdy SC, Cliby WA, et al. Multicenter study comparing oncologic outcomes between two nodal assessment methods in patients with deeply invasive endometrioid endometrial carcinoma: A sentinel lymph node algorithm versus a comprehensive pelvic and paraaortic lymphadenectomy. Gynecol Oncol 2018; 151:235-242. doi: 10.1016/j.ygyno.2018.08.022. [PubMed: 30177461]

18. Schiavone MB, Zivanovic O, Zhou Q, Leitao MM Jr, Levine DA, Soslow RA, et al. Survival of Patients with Uterine Carcinosarcoma Undergoing Sentinel Lymph Node Mapping. Ann Surg Oncol 2016; 23:196-202. doi: 10.1245/s10434-015-4612-2. [PubMed: 25994210]

19. Touhami O, Grégoire J, Renaud MC, Sebastianelli A, Plante M. Performance of sentinel lymph node (SLN) mapping in high-risk endometrial cancer. Gynecol Oncol 2017; 147:549-553. doi: 10.1016/j.ygyno.2017.09.014. [PubMed: 28942993]

20. Todo Y, Kato H, Kaneuchi M, Watari H, Takeda M, Sakuragi N. Survival effect of para-aortic lymphadenectomy in endometrial cancer (SEPAL study): a retrospective cohort analysis. Lancet 2010; 375:1165-1172. doi: 10.1016/S0140-6736(09)62002-X. [PubMed: 20188410] 
21. Venigalla S, Chowdhry AK, Shalowitz DI. Survival implications of staging lymphadenectomy for non-endometrioid endometrial cancers. Gynecol Oncol 2018; 149:531-538. doi: 10.1016/j.ygyno. 2018.03.046 [PubMed: 29559170] 


\section{Highlights}

- $\quad$ Systematic LND showed no survival benefit vs SLN mapping alone in patients with USC

- Detection of nodal metastasis is the same in SLN mapping alone vs systematic LND

- $\quad$ Advanced disease and LVSI were the most significant factors in oncologic outcomes 


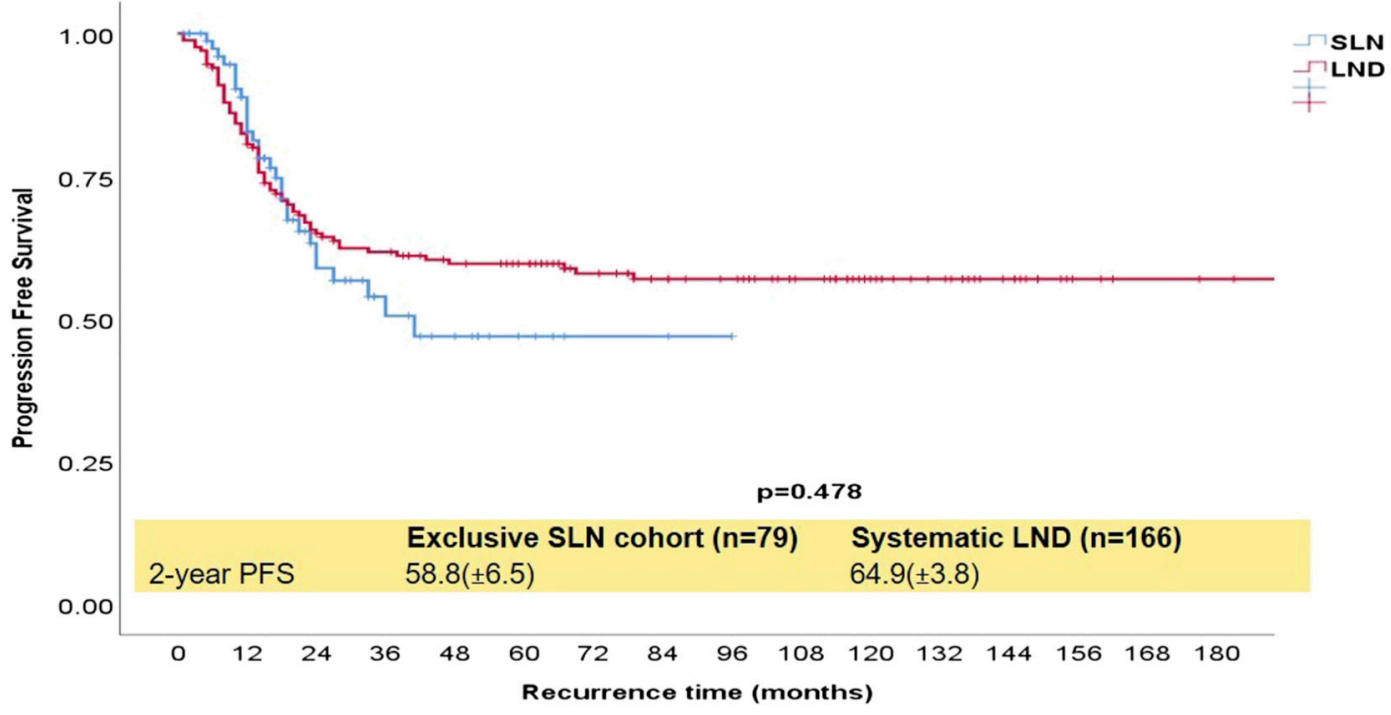

Figure 1.

Progression-free survival for all patients with USC 


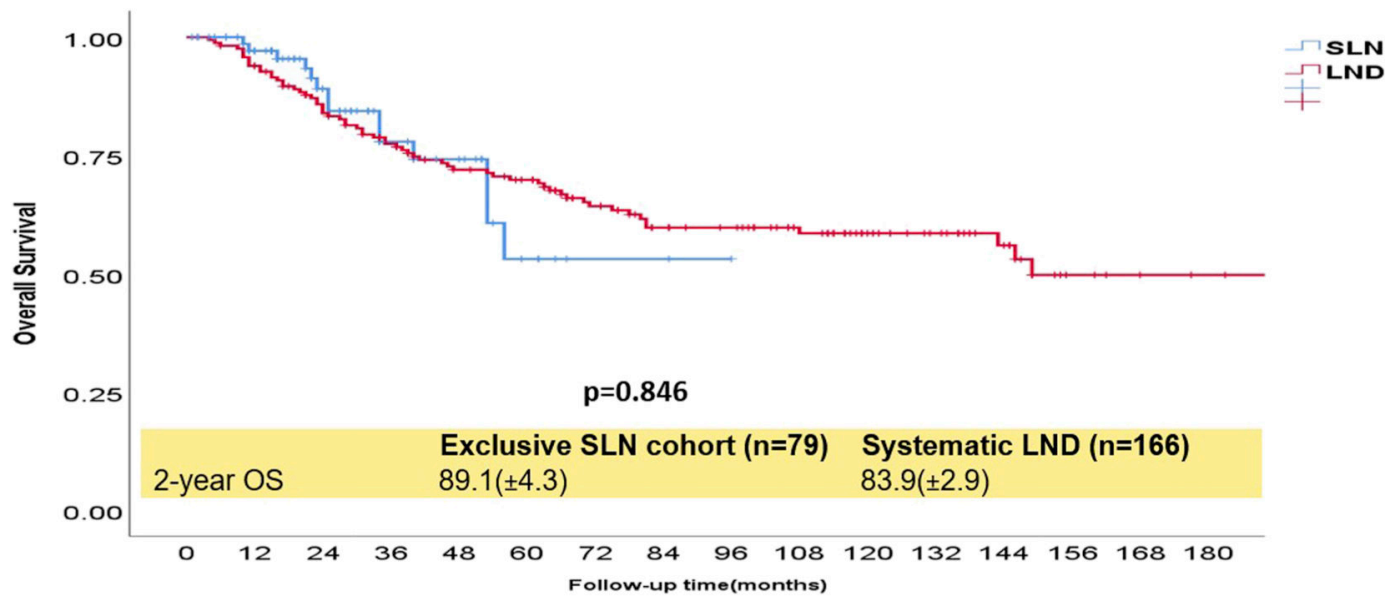

Figure 2.

Overall survival for all patients with USC 


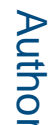 \\ 蝗}



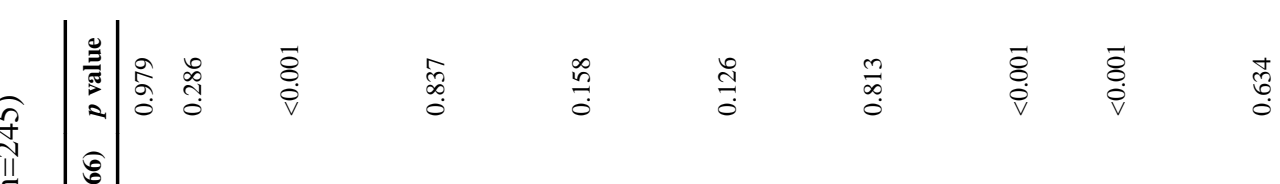

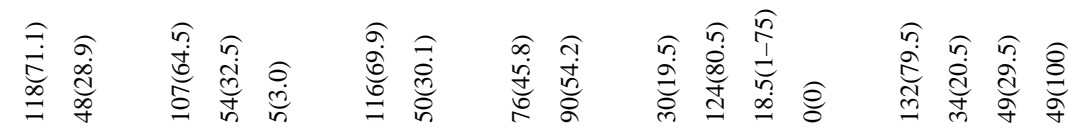

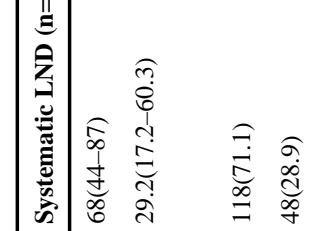
点 造

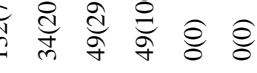

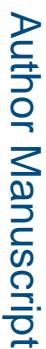

ग

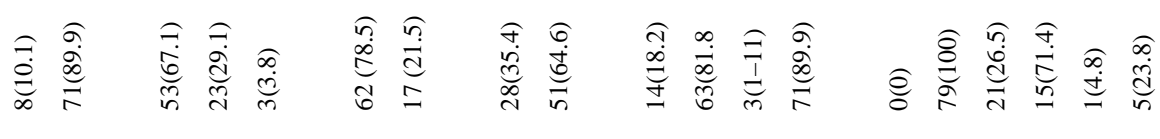

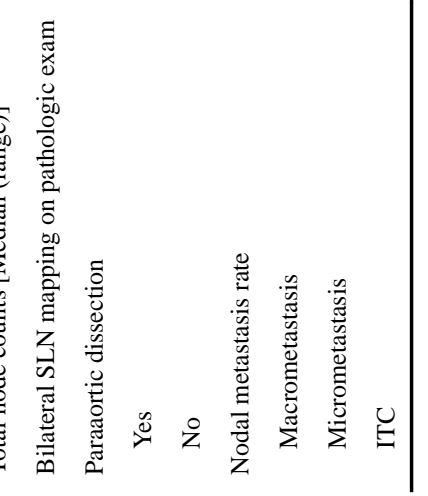




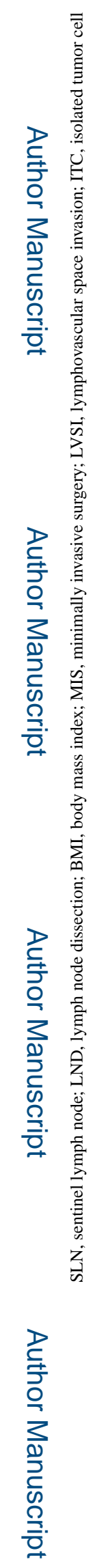




\section{를}

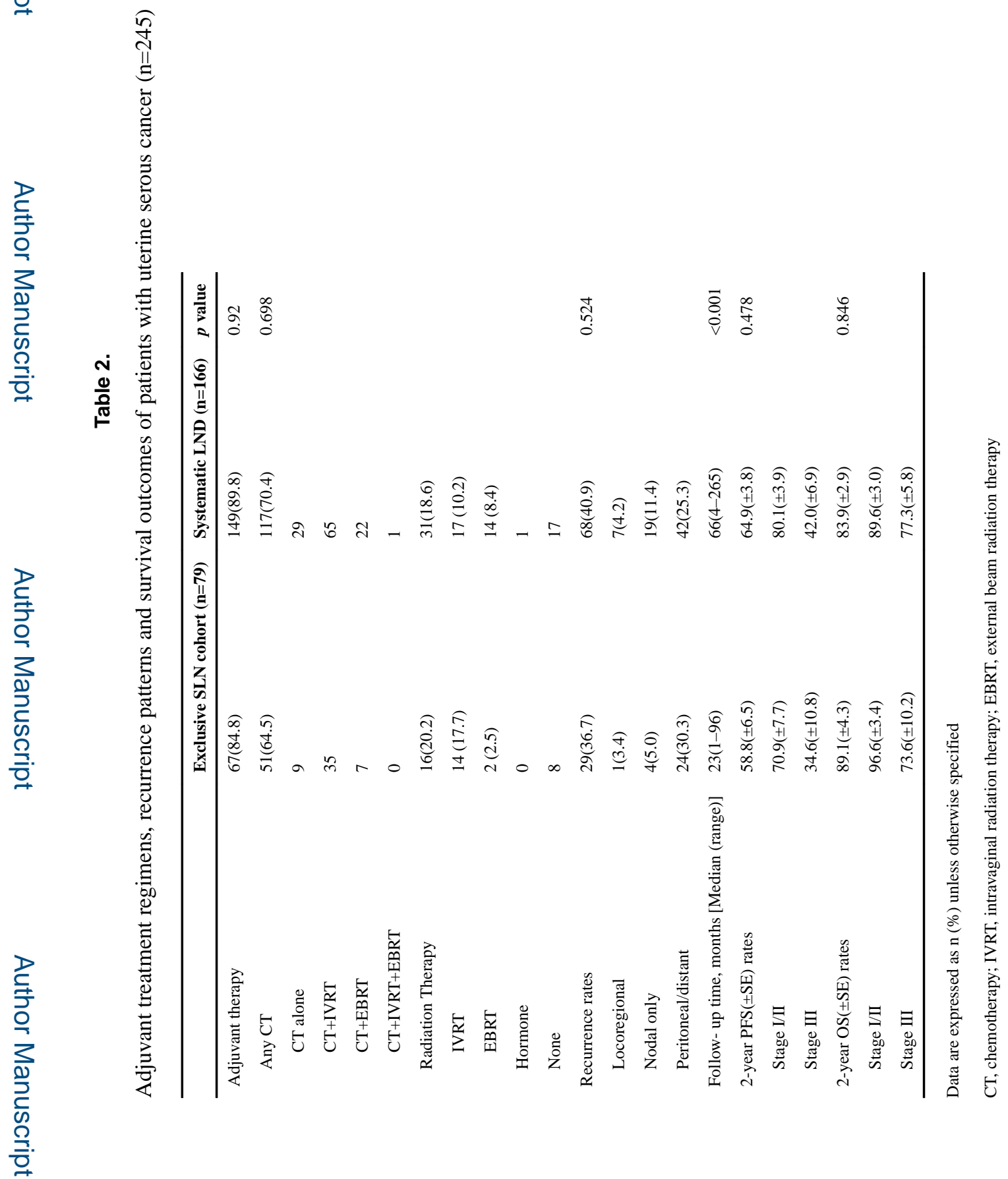

Gynecol Oncol. Author manuscript; available in PMC 2021 January 01. 


\section{롤 \\ 골}

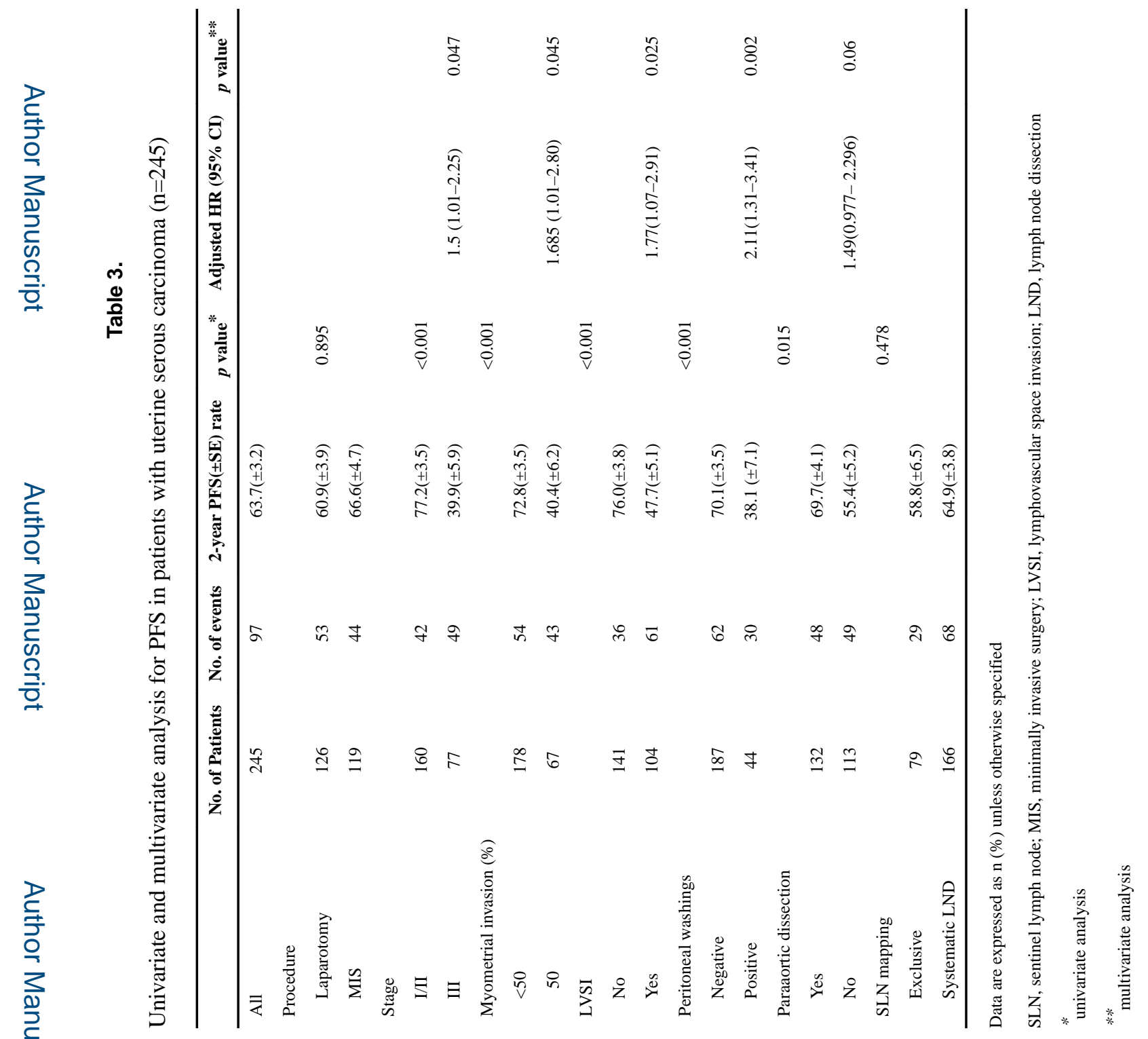

Gynecol Oncol. Author manuscript; available in PMC 2021 January 01. 

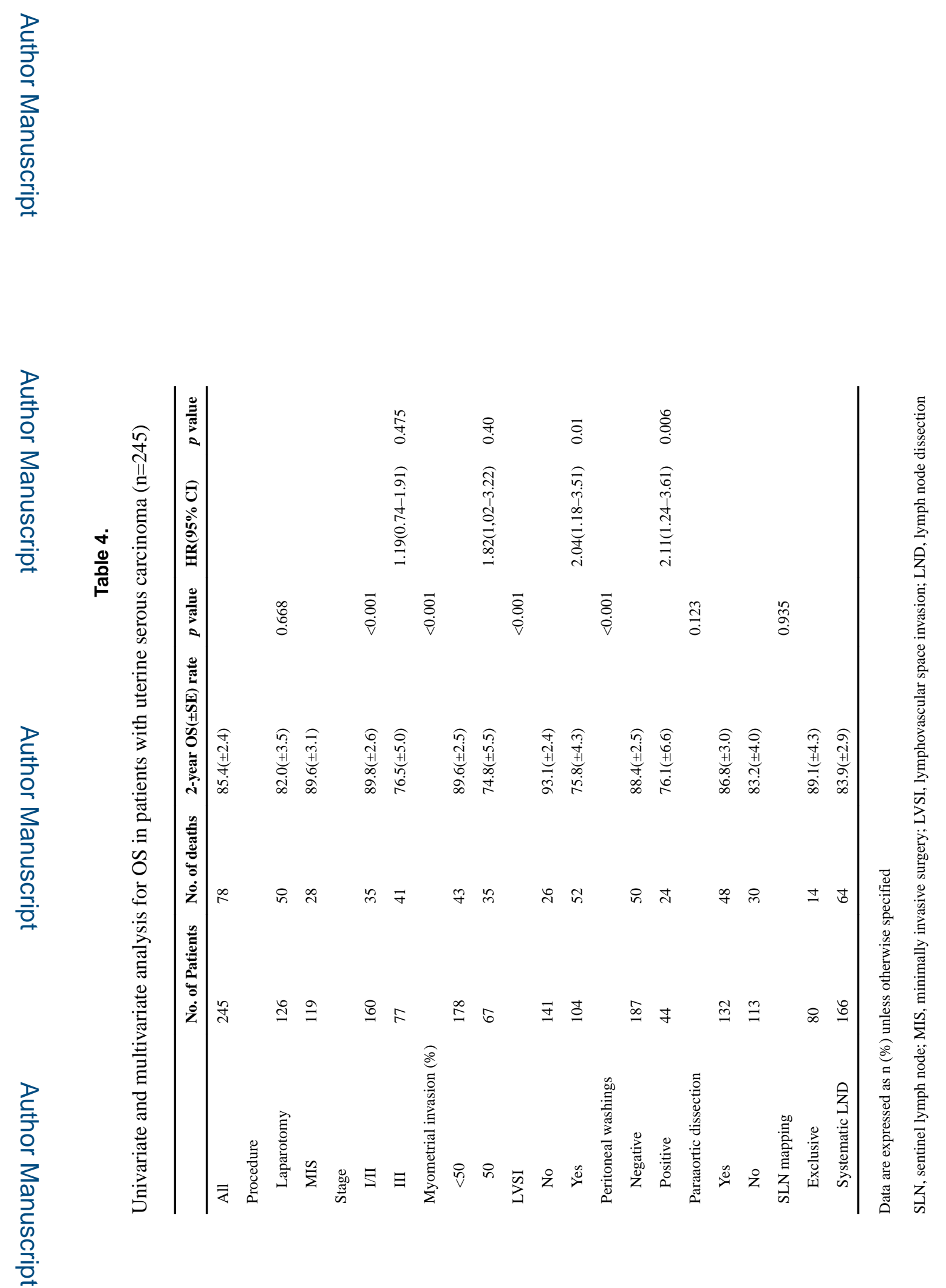

Gynecol Oncol. Author manuscript; available in PMC 2021 January 01. 